\title{
Systematic Search for Halos Around Pulsars in Fermi-LAT Data
}

\author{
Soheila Abdollahi ${ }^{a, *}$, Pierrick Martin $^{a}$, Luigi Tibaldo $^{a}$, Jürgen Knödlseder ${ }^{a}$, and \\ Mattia Di Mauro ${ }^{b}$ on behalf of the Fermi Large Area Telescope Collaboration \\ a IRAP, Université de Toulouse, CNRS, UPS, CNES, \\ F-31028, Toulouse, France \\ ${ }^{b}$ Istituto Nazionale di Fisica Nucleare, Sezione di Torino, \\ via P. Giuria, 1, 10125, Torino, Italy \\ E-mail: soheila.abdollahi@irap.omp.eu, pierrick.martin@irap.omp.eu
}

Extended gamma-ray halos around middle-aged pulsars, discovered at TeV energies from HAWC observations, are a new and potentially rich source class. The phenomenon is interpreted as the inverse-Compton scattering of ambient photons by electron/positron pairs accelerated by pulsars and their nebulae and confined in their vicinity. Physically, the dynamics of this pair confinement remains poorly understood and halos offer an opportunity to probe the neighborhood of pulsar/supernova remnant systems, e.g., the magnetic field structure and conditions for energetic particle transport. As a population, due to their large sizes and long lifetimes, halos can be expected to have a non-negligible contribution to the $\mathrm{GeV}-\mathrm{TeV}$ emission from the Galaxy, in the form of currently unidentified sources and/or unresolved emission on large scales. In the GeV range, the first detections of halo candidates were achieved recently and the phenomenon essentially remains to be explored. We have performed a systematic search for halos around middle-aged pulsars using 12.8 years of Fermi-LAT data. We have set up an analysis suite to detect extended emission around a selection of ATNF pulsars likely to harbor halos at a detectable level. We present a list of promising halo candidates, together with dedicated studies in which we investigate the morphology and spectrum of a few selected targets. Combined with measurements at other wavelengths, this provides information on the physical processes underlying the formation of pulsar halos and contributes to a better assessment of halos as a population.

$3^{\text {th }}$ International Cosmic Ray Conference (ICRC 2021)

July 12th - 23rd, 2021

Online - Berlin, Germany

\footnotetext{
*Presenter
} 


\section{Introduction}

The High-Altitude Water Cherenkov Observatory (HAWC), building upon previous reports by Milagro, revealed a number of extended $\mathrm{TeV} \gamma$-ray sources surrounding nearby middle-aged pulsars such as Geminga and PSR B0656+14 [1-3]. Subsequent observations grew the population of extended $\mathrm{TeV}$ emission coincident with pulsars or pulsar wind nebulae (PWNe) and suggested the existence of a new class of objects in the $\gamma$-ray sky, dubbed " $\gamma$-ray halos" [4]. The halo emission is attributed to the cosmic-ray (CR) electrons/positrons that diffuse away from the pulsar/PWN system and interact with ambient interstellar radiation fields. The level and distribution of the emission suggest that pairs are efficiently confined in a region with significantly inhibited diffusion compared to the surrounding interstellar medium (ISM), although alternative explanations were recently put forward [5]. Halos have been suggested as a non-negligible contribution to the Galactic diffuse $\gamma$-ray emission [6], and some models predict that more than half of the diffuse emission currently attributed to TeV halos will be resolved into individual sources in the future [7].

Despite a large number of studies on halos in the $\mathrm{TeV}$ domain, their properties at $\mathrm{GeV}$ energies remain to be explored. In this proceeding, we perform the first systematic search for $\mathrm{GeV}$ halos around pulsars using $\sim 13$ years of Fermi-LAT data. We provide a list of pulsars extracted from the Australian Telescope National Facility (ATNF) pulsar catalog [8] that are likely to be detectable as $\mathrm{GeV}$ halos as predicted by our model. Of the selected 289 sources from the ATNF catalog, 13 that met our selection criteria are analyzed. A discussion on the population of $\mathrm{GeV}$ halos along with their morphological and spectral properties is presented.

\section{Selection of Candidate Pulsars}

A total of 289 sources with characteristic age $\tau_{\mathrm{c}}>60 \mathrm{kyr}$, spin-down power $\dot{E}>10^{33} \mathrm{erg} \mathrm{s}^{-1}$, and distance $d<10 \mathrm{kpc}$ were extracted from the ATNF catalog. A two-zone diffusion model similar to that applied to the Geminga halo in [9] was implemented and used for the full sample. A constant fraction of the declining spin-down power of the pulsar is converted into high-energy pairs with a spectrum consisting of a broken power-law with low-energy index $\gamma_{1}=1.5$, high-energy index $\gamma_{2}=2.3$, and a break energy $E_{\mathrm{b}}=100 \mathrm{GeV}$. Injection into the halo starts at $t>60 \mathrm{kyr}$, after the typical duration of the PWN stage. The propagation of particles away from the pulsar and into the ISM is assumed to proceed from diffusion, in a two-zone model where diffusion is suppressed within $50 \mathrm{pc}$ of the pulsar by a factor of hundreds compared to the typical ISM. Inside the low diffusion region, the rigidity-dependent diffusion coefficient has the form $D \sim D_{0} \beta R_{\mathrm{TV}}^{\delta} \mathrm{cm}^{2} \mathrm{~s}^{-1}$, with $\delta=1 / 3$ typical of Kolmogorov diffusion and a normalization $D_{0}=2.3 \cdot 10^{27} \mathrm{~cm}^{2} \mathrm{~s}^{-1}$ at $100 \mathrm{TeV}$, consistent with the value inferred from HAWC observations of Geminga and B0656+14 [2]. Along their propagation, pairs experience energy losses, mostly from synchrotron and inverseCompton scattering in interstellar magnetic and radiation fields, respectively. Galactic models for the latter were obtained from [10], as a double exponential model with $R_{\mathrm{B}}=6 \mathrm{kpc}, z_{\mathrm{B}}=2 \mathrm{kpc}$, and $B_{0}=3 \mu \mathrm{G}$, and [11]. Eventually, an angular distribution of inverse-Compton (IC) emission is computed from the three-dimensional distribution of pairs around the pulsar.

Based on the predicted halo emission for the 289 pulsars, we selected a subset meeting the following criteria: i) predicted halo $68 \%$ containment radius $r_{68}<1^{\circ}$ in the $10-100 \mathrm{GeV}$ range, in order to minimize confusion with the Galactic diffuse emission and nearby spatially extended sources if 


\begin{tabular}{lllccccc}
\hline \hline PSR Candidate & 4FGL Source & Class & $\begin{array}{c}l \\
\left({ }^{\circ}\right)\end{array}$ & $\begin{array}{c}b \\
\left({ }^{\circ}\right)\end{array}$ & $\begin{array}{c}d \\
(\mathrm{kpc})\end{array}$ & $\begin{array}{c}\tau_{\mathrm{c}} \\
(\mathrm{kyr})\end{array}$ & $\begin{array}{c}\dot{E} \\
\left(10^{36} \mathrm{erg} / \mathrm{s}\right)\end{array}$ \\
\hline PSR B1951+32 & 4FGL J1952.9+3252 & PSR & 68.77 & 2.82 & 3.00 & 107 & 3.74 \\
PSR J1913+1011 & 4FGL J1913.3+1019 & PSR & 44.49 & -0.17 & 4.61 & 169 & 2.87 \\
PSR J1928+1746 & 4FGL J1928.4+1801c & unk & 52.93 & 0.11 & 4.34 & 82.6 & 1.60 \\
PSR J1437-5959 & 4FGL J1435.8-6018 & spp & 315.78 & 0.23 & 8.54 & 114 & 1.44 \\
PSR J1101-6101 & 4FGL J1102.0-6054 & spp & 290.04 & -0.93 & 7.00 & 116 & 1.36 \\
PSR J1718-3825 & 4FGL J1718.2-3825 & PSR & 348.95 & -0.43 & 3.49 & 89.5 & 1.25 \\
PSR J1925+1720 & 4FGL J1925.2+1729 & PSR & 52.18 & 0.59 & 5.06 & 115 & 0.95 \\
PSR J1028-5819 & 4FGL J1028.5-5819 & PSR & 285.07 & -0.50 & 1.42 & 90 & 0.83 \\
PSR B1259-63 & 4FGL J1303.0-6312e & PWN & 304.18 & -0.99 & 2.63 & 332 & 0.83 \\
PSR J1151-6108 & 4FGL J1152.0-6107 & PSR & 295.81 & 0.91 & 2.22 & 157 & 0.39 \\
PSR J1740+1000 & 4FGL J1740.5+1005 & PSR & 34.01 & 20.27 & 1.23 & 114 & 0.23 \\
PSR B0114+58 & 4FGL J0102.8+5824 & fsrq & 126.28 & -3.46 & 1.77 & 275 & 0.22 \\
PSR B0740-28 & 4FGL J0742.8-2822 & PSR & 243.77 & -2.44 & 2.00 & 157 & 0.14 \\
\hline PSR B1823-13 & 4FGL J1824.5-1351e & PWN & 18.00 & -0.69 & 3.61 & 21.4 & 2.84 \\
PSR J1907+0602 & 4FGL J1907.9+0602 & PSR & 40.18 & -0.89 & 2.37 & 19.5 & 2.83
\end{tabular}

Table 1: Properties of halo candidates that fulfill the selection criteria. The associated 4FGL sources and their classes are given in the second and third column. The last two candidates are the young pulsars with recently reported halo around them [12].

there are any, ii) mature pulsars with characteristic age $\tau_{\mathrm{c}}>80 \mathrm{kyr}$, to focus on objects that are well passed the PWN stage, and iii) $d>1 \mathrm{kpc}$, to be able to neglect the angular displacement due to the pulsar proper motion, and iv) halos with photon flux $>20 \%$ of the interstellar diffuse emission, computed in the energy range $10-100 \mathrm{GeV}$ and within $68 \%$ containment radius around the position of pulsars, and using the standard Galactic diffuse emission model (gll_iem_v07. fits), provided by the Fermi-LAT collaboration.

These criteria lead to 14 middle-aged candidates as listed in Table 1. One of the candidates, PSR J0855-4644, associated with supernova remnant RX J0852.0-4622 (Vela Jr), is excluded from the list due to the complexity of the surrounding region. Two young pulsars ( $t \sim 20 \mathrm{kyr}$ ), recently claimed to be associated with halo-like extended emission [12], are included in the list to perform sanity checks on our analysis procedure.

\section{Fermi-LAT Data Selection}

In the following, we analyze $\sim 154$ months of the Fermi-LAT data (collected from August 4, 2008 to May 4, 2021) employing a dedicated pipeline based on the Fermipy Python package $^{1}$ (version 1.0.1) and the standard Fermitools ${ }^{2}$ (version 2.0.8), to systematically search for $\mathrm{GeV}$ halos around our selected set of targets. The event selection is based on the low background Pass 8 SOURCEVETO event class (FRONT+BACK) and the corresponding instrument response functions is P8R3_SOURCEVETO_V3, which is best suited for diffuse emission analysis. A zenith angle

1https://github.com/Fermipy/fermipy

2https://fermi.gsfc.nasa.gov/ssc/data/analysis/software/ 
cut of $105^{\circ}$ is applied to minimize the contribution of the Earth limb [13] and the standard filter (DATA_QUAL $>0) \& \&\left(L A T \_C O N F I G==1\right.$ ) is used. In this analysis, we selected photons within a square region of interest (RoI) of side $7^{\circ}$ centered on each target. Our source models contain all 4FGL sources within a region of $13^{\circ}$ around each target, along with the standard Galactic diffuse emission model gll_iem_v07.fits and isotropic template iso_P8R3_SOURCEVETO_V3_v1.txt. ${ }^{3}$ The broader source region is to account for any possible contamination from outside the RoI. The energy dispersion correction is applied to all components of the sources model but the Galactic diffuse and isotropic components. We performed a maximum-likelihood analysis for binned data in the energy range $10 \mathrm{GeV}-1 \mathrm{TeV}$, using 5 logarithmic energy bins per decade and $0.05^{\circ}$ spatial pixels. The $10 \mathrm{GeV}$ energy threshold is meant to allow easier separation of halos from the Galactic interstellar diffuse emission. Source significance for all sources in the RoI is quantified via the Test Statistic TS $=2 \ln \left(\mathcal{L} / \mathcal{L}_{0}\right)$ [14], where $\mathcal{L}$ and $\mathcal{L}_{0}$ represent the maximum likelihood value for an emission model with and without the test source, respectively. The next section outlines the analysis procedure we applied to all 15 targets, independently.

\section{Analysis Method}

The analysis starts with an initial optimization of each RoI, iteratively, in three steps: i. fitting the normalization of maximum five sources with the largest number of expected counts that together amount to more than $95 \%$ of the total number predicted counts, ii. fitting individually the normalization of the rest of sources in the model, those with predicted photons of at least 1.0, iii. fitting individually the normalization and spectral parameters of all model sources with TS $>25$.

An inherent challenge to search for extended emission around sources is possible confusion with nearby faint sources with a TS below the detection threshold, and the influence of other extended components such as the isotropic emission. In order to properly address this problem, several approaches were investigated and we retained the one that yields the best Akaike information criterion (AIC; [15] given by AIC $=2 k-2 \ln \mathcal{L}$ where $k$ was the number of fit parameters in the model) for the largest number of targets in our list. Eventually, dim sources with $\mathrm{TS}<25$ are fixed at their best-fit value after the initial optimization, and the isotropic is left free to vary. A global fit was performed to adjust the following free parameters: i) normalization of sources with TS between 25 and 50 within $2^{\circ}$ of the RoI center, ii) normalization and spectral parameters of sources with $\mathrm{TS}>50$ in the same zone, iii) normalization of sources with TS $>25$ between $2^{\circ}$ and $3^{\circ}$ of the RoI center, iv) normalization of the Galactic and isotropic diffuse emission models. Moreover, strong sources with TS $>500$ were left to vary, regardless of their distance from the center of RoI. The resulting set of free parameters is kept for any global fit throughout the analysis. The AIC value of the model at this step was defined as a reference $\left(\mathrm{AIC}_{\mathrm{ref}}\right)$ in the rest of analysis.

We then searched for any possible extension emission around the central pulsar. Two independent hypotheses were tested to find the best model for the central source as follow:

(i) We performed an extension analysis for the central source by computing the likelihood ratio of the source with a $2 \mathrm{D}$ Gaussian morphology ( $\mathcal{L}_{\text {ext }}$, as a test hypothesis) with respect to the point-like hypothesis ( $\mathcal{L}_{\mathrm{ps}}$, as a null hypothesis), and then applied a localization procedure to update the source position.

\footnotetext{
3https://fermi.gsfc.nasa.gov/ssc/data/access/lat/BackgroundModels.html
} 
(ii) We tested a model in which there is an additional component with a 2D Gaussian morphology and a power-law spectrum of spectral index 2.3 on top the central source, here again optimizing for extension and then position.

After comparison of the AIC of these two tests and the reference one $\left(\mathrm{AIC}_{\mathrm{ref}}\right)$, the model with the lowest AIC value is adopted as the best model for the following. At this stage, new sources as point sources with TS $>25$ and extended sources with a 2D Gaussian morphology, TS $>25$ and $\mathrm{TS}_{\mathrm{ext}}=2 \ln \left(\mathcal{L}_{\text {ext }} / \mathcal{L}_{\mathrm{ps}}\right)>16$ were searched for in the RoI and included in a global fit. In the last stage, the best model for the central source, now possibly including a halo component, is revisited, following the same procedure explained above, to refine its spatial and spectral parameters after identifying new sources in the RoI.

In the next section, we present the results of our search on $\mathrm{GeV}$ halos utilizing this procedure.

\section{Fermi-LAT Halo Candidates}

Out of the 15 sources presented in Table 1, we detected additional significantly extended emission for 9 of them ( 7 middle-aged candidates and the 2 young ones) with $\mathrm{TS}_{\mathrm{ext}}>16$. The other 6 sources fall below our threshold for extended emission ( 5 of them with $\mathrm{TS}_{\mathrm{ext}}<1$, and PSR B1951+32 with $\mathrm{TS}_{\mathrm{ext}}=13$ ). The optimized position and extension of the halo candidates along with their angular and physical offsets and their significance are summarized in Table 2. Here, we have presented the significance of the halo candidate $\left(\mathrm{TS}_{\text {halo }}\right)$, and of the complete system including both halo candidate and central source $\left(\mathrm{TS}_{\text {sys }}\right)$. Figure 1 shows the significance maps of 6 significant middle-aged halo candidates. Since these significance maps are only sensitive to positive excesses, we checked the residual counts maps to ensure there is no significant negative excess in the residual counts maps. The spectral energy distributions of these candidates as well as two young ones in the energy range $10 \mathrm{GeV}-1 \mathrm{TeV}$ are shown in Figure 2. All sources have rather hard spectrum index with significant flux above $100 \mathrm{GeV}$. The statistical upper limits are calculated at $95 \%$ confidence level from the profile likelihood when the detection is not significant $(\mathrm{TS}<4)$ [16].

Our measurements for the two young candidates are in good agreement with a recent study [12]. The small differences in the results for PSR B1823-13 can be explained by the energy-dependent morphology of the system, that we cannot handle with our energy-independent extended emission models, and the fact that a component for the PWN was included in our analysis, so we look for emission on top and beyond the PWN, and do not absorb all of it in a single 2D Gaussian. Our detection of extended emission around PSR J1913+1011, in the vicinity of TeV source 2HWC J1912+099 and spatially coincident with HESS 1912+101, confirms the results of [17]. Although PSRs J1928+1746 and J1925+1720 are very close on the sky ( $0.89^{\circ}$ separation), the extended emissions detected around them are clearly distinct in both position and extension. Nevertheless, more robust results avoiding a possible cross-talk between the two overlapping component and other extended component deserves a joint analysis that is deferred to a further publication, and for this reason the significance map of pulsar J1925+1720 is not shown in Figure 1. PSR J1928+1746 is especially

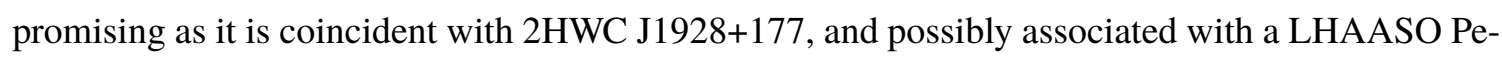
Vatron candidate, LHAASO J1929+1745 [18]. Last, the highest-significance halo candidate in our list is found around PSR J1028-5819, that was previously reported as a potential PWN candidate at $4 \sigma$ level [19] and is positionally coincident with the TeV source HESS J1026-582 [20]. Among the detected extended emission around 7 middle-aged pulsars, only J1028-5819 has overlapped 
with a 4FGL extended source (4FGL J1036.3-5833e). The detected extended emission around PSR $\mathrm{J} 1028-5819$ has the same angular size as the 4FGL source and the offset between them is $0.17^{\circ}$. The detected halo candidate slightly better models the diffuse emission around the pulsar compared to the initial 4FGL extended source. Two other halo candidates, J1718-3825 and B1259-63, partially overlap with the 4FGL extended sources 4FGL J1713.5-3945e and 4FGL J1303.0-6312e, respectively. For the rest 4 halo candidates, the surrounding regions are clean of 4FGL extended sources.

\begin{tabular}{lccccccc}
\hline \hline \multirow{2}{*}{ PSR Candidate } & $l$ & $b$ & $r_{68}$ & \multicolumn{2}{c}{ Offset } & \multirow{2}{*}{$\mathrm{TS}_{\text {halo }}$} & $\mathrm{TS}_{\text {sys }}$ \\
\hline PSR J1028-5819 & $285.92 \pm 0.08$ & $-0.14 \pm 0.07$ & $2.02 \pm 0.14$ & 0.92 & 23 & 370 & 1165 \\
PSR J1913+1011 & $44.68 \pm 0.14$ & $-0.10 \pm 0.09$ & $0.79 \pm 0.09$ & 0.20 & 16 & 47 & 123 \\
PSR J1928+1746 & $53.49 \pm 0.21$ & $0.11 \pm 0.15$ & $1.34 \pm 0.03$ & 0.56 & 42 & 39 & 44 \\
PSR J1925+1720 & $51.28 \pm 0.05$ & $0.27 \pm 0.08$ & $0.34 \pm 0.07$ & 0.95 & 84 & 41 & 42 \\
PSR J1101-6101 & $289.45 \pm 0.15$ & $-0.64 \pm 0.20$ & $0.61 \pm 0.12$ & 0.66 & 81 & 21 & 26 \\
PSR J1718-3825 & $347.76 \pm 0.30$ & $-0.93 \pm 0.25$ & $1.70 \pm 0.33$ & 1.29 & 79 & 38 & 58 \\
PSR B1259-63 & $304.92 \pm 0.18$ & $-0.08 \pm 0.11$ & $0.74 \pm 0.10$ & 1.17 & 54 & 30 & 258 \\
\hline PSR B 1823-13 & $17.72 \pm 0.14$ & $-0.34 \pm 0.14$ & $1.98 \pm 0.17$ & 0.46 & 29 & 66 & 592 \\
PSR J1907+0602 & $40.49 \pm 0.12$ & $-0.65 \pm 0.12$ & $0.74 \pm 0.09$ & 0.39 & 16 & 48 & 477 \\
\hline
\end{tabular}

Table 2: Best-fit spatial information and TS values of the halo candidates around pulsars. The $\mathrm{TS}_{\mathrm{sys}}$ corresponds to the TS of both the halo candidates and the central source. The offset of the halo candidates with respect to the target pulsars in terms of both angular and physical distance are given. The last two sources are the young pulsars.

\section{Conclusions}

In a systematic search for $\mathrm{GeV}$ halos around middle-aged pulsars using 12.8 years of FermiLAT data, 7 out of 13 middle-aged candidates revealed significant extended emission around the pulsars. Interestingly, the positional offsets from the pulsars and the extensions of these additional components correspond to physical sizes in the range $50-100 \mathrm{pc}$, as expected for low-energy Geminga-like halos developing around mature pulsars possibly animated by proper motions at a few $100 \mathrm{~km} \mathrm{~s}^{-1}$. All halo candidates exhibit pretty hard emission spectra reaching beyond $100 \mathrm{GeV}$, which is also expected in the context of inverse-Compton halos from a population of very-highenergy pairs. The results presented here are encouraging and further demonstrates that Fermi-LAT can contribute to the growing knowledge on this emerging class of sources. More work is however needed to ascertain that these extended components do not simply result from, or are not strongly biased by, imperfections in the modeling of the Galactic diffuse emission.

\section{Acknowledgements}

The Fermi-LAT Collaboration acknowledges support for LAT development, operation and data analysis from NASA and DOE (United States), CEA/Irfu and IN2P3/CNRS (France), ASI and INFN (Italy), MEXT, KEK, and JAXA (Japan), and the K.A. Wallenberg Foundation, the Swedish Research Council and the National Space Board (Sweden). Science analysis support in the 

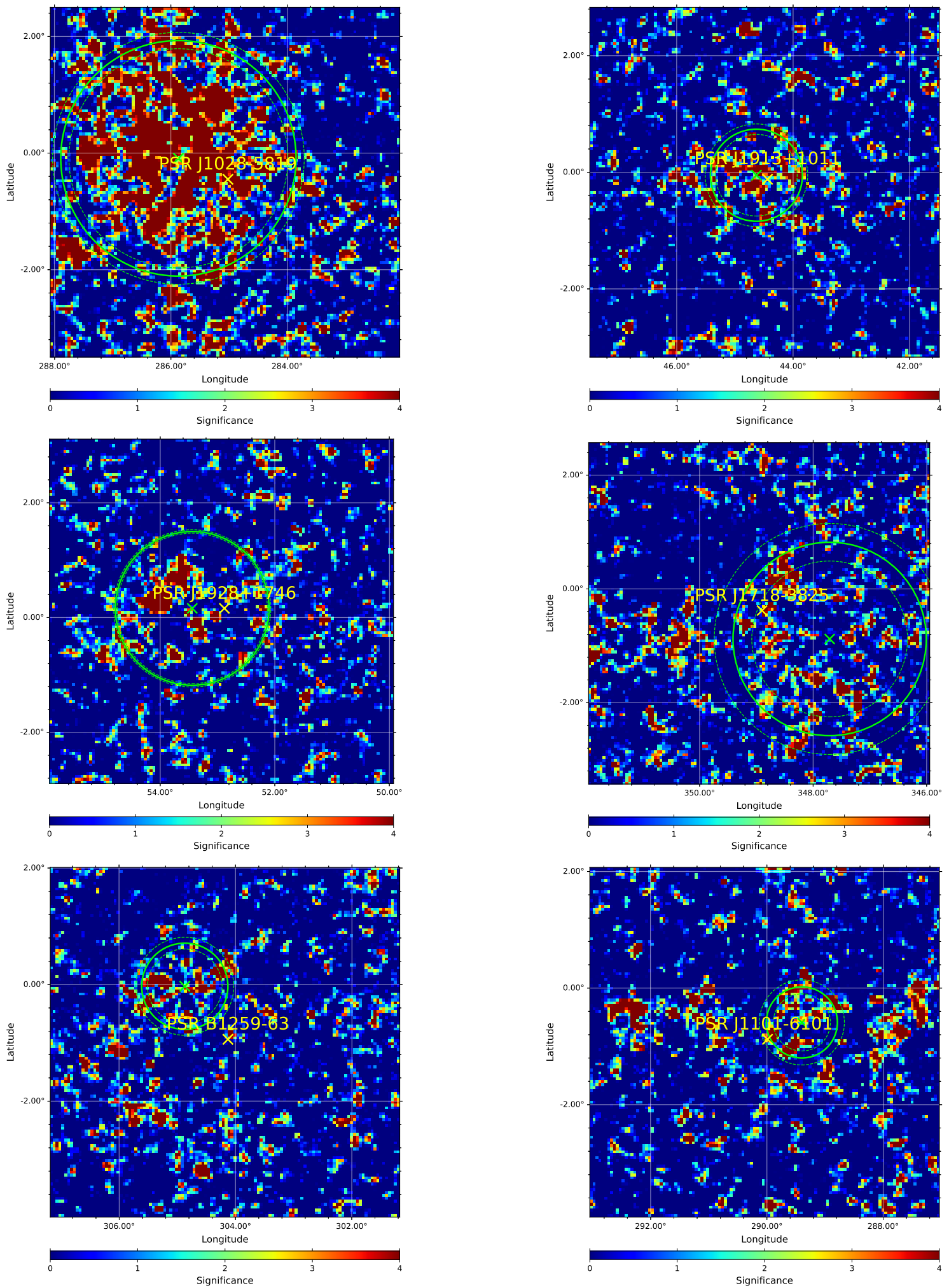

Figure 1: Significance maps of 6 out of 7 significant middle-aged halo candidates in the energy range 10-1000 GeV. The detected extended emission are excluded from their models. The green crosses depict the best-positions of the halo candidates. The $68 \%$ containment radii of the halo candidates obtained by the Gaussian model are shown with green circles and dashed circles indicate their inner and outer radii. The yellow crosses represent the positions of the central pulsars. 


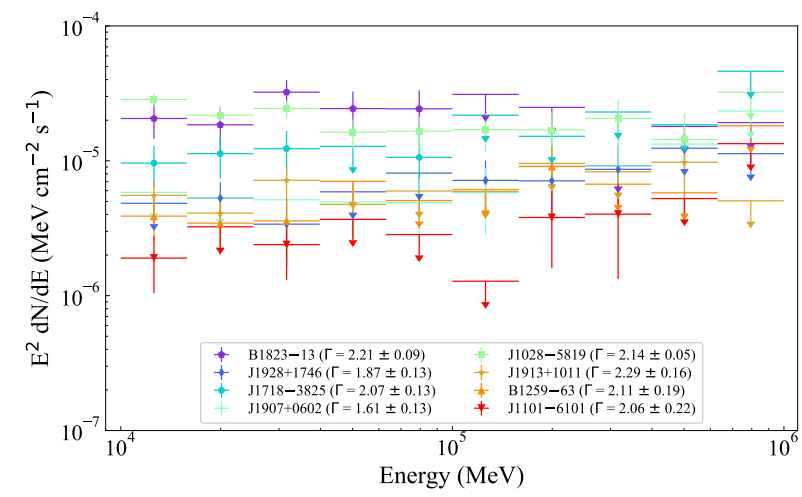

Figure 2: Spectral energy distribution of $\mathrm{GeV}$ halo candidates. The best-fit values of the spectral index $\Gamma$ are displayed in parentheses in the legend.

operations phase from INAF (Italy) and CNES (France) is also gratefully acknowledged. This work performed in part under DOE Contract DE-AC02-76SF00515. We acknowledge financial support by CNES for the exploitation of Fermi-LAT observation. We acknowledge financial support from the French Agence Nationale de la Recherche under reference ANR-19-CE31-0014 (GAMALO project).

\section{References}

[1] Abdo, A. A., Allen, B. T., Aune, T., et al. 2009, ApJ, 700, L127

[2] Abeysekara, A. U., Albert, A., Alfaro, R., et al. 2017, Science, 358, 911

[3] Abeysekara, A. U., Albert, A., Alfaro, R., et al. 2017, ApJ, 843, 40

[4] Linden, T., Auchettl, K., Bramante, J., et al. 2017, PRD, 96, 103016

[5] Recchia, S., Galli, D., Nava, L., et al. 2021, arXiv:2106.04948v1 [astro-ph.HE]

[6] Cataldo, M., Pagliaroli, G., Vecchiotti, V., and Villante, F. .L. 2020, ApJ, 904, 85

[7] Sudoh, T., Linden, T., and Beacom, J. F. 2019, PRD, 100, 043016

[8] Manchester, R. N., Hobbs, G. B., Teoh, A., and Hobbs, M. 2005, AJ, 129, 1993

[9] Tang, X. \& Piran, T. 2019, MNRAS, 484, 3491

[10] Evoli, C., Gaggero, D., Vittino, A., et al. 2017, JCAP, 02, 015

[11] Popescu, C. C., Yang, R., Tuffs, R. J., et al. 2017, MNRAS, 470, 2539

[12] Di Mauro, M., Manconi, S., Negro, M., and Donato, F. 2020, arXiv:2012.05932 [astro-ph.HE]

[13] Abdo, A. A., Ackermann, M., Ajello, M., et al. 2009, ApJS, 183, 46

[14] Mattox, J. R., Bertsch, D. L., Chiang, J., et al. 1996, ApJ, 461, 396

[15] Akaike, H. 1974, IEEE Trans. Autom. Control, 19, 716

[16] Rolke, W. A., López, A. M., Conard, J. 2005, NIM A 551, 493

[17] Zhang, H.-M., Xi, Sh.-Q., Liu, R.-Y, et al. 2020, ApJ, 889, 12

[18] Cao, Z., Aharonian, F. A., An, Q., et al. 2021, Nature 594, 33

[19] Ackermann, M., Ajello, M., Baldini, L., et al. 2011, ApJ, 726, 35

[20] Abramowski, A., Acero, F., Aharonian, F., et al. 2011, A\& A, 525, A46 